Supporting Information for:

\title{
The MPEG effect: Improving asymmetric processes by simple additives
}

\author{
Jens Rudolph, Nina Hermanns and Carsten Bolm* \\ Institut für Organische Chemie der RWTH Aachen, Professor \\ Pirlet Strasse 1,D-52056 Aachen, Germany. \\ carsten.bolmeoc.rwth-aachen.de
}

\section{Table of Contents:}

- analytical data of compounds 3 and 6;

- HPLC separation conditions for compounds 3, 6, 7 .

- References

- ${ }^{1} \mathrm{H}$ - und ${ }^{13} \mathrm{C}-\mathrm{NMR}$ spectra for compounds $3, \mathbf{6 ,} \mathbf{7}$.

\section{Analytical data}

\section{4-Chlorophenyl-phenylmethanol (3) ${ }^{1}$}

Compound 3 was obtained as a white solid with up to 95\% yield ( $52 \mathrm{mg}, 0.24 \mathrm{mmol}$ ).

${ }^{1} \mathrm{H} \operatorname{NMR}\left(\mathrm{CDCl}_{3}, 400 \mathrm{MHz}\right): \delta=2.23(\mathrm{sbr}, 1 \mathrm{H}, \mathrm{OH}), 5.78(\mathrm{~s}, 1 \mathrm{H}$, $\mathrm{CH}), 7.23-7.45\left(\mathrm{~m}, 9 \mathrm{H}, \mathrm{H}_{\mathrm{ar}}\right)$.

${ }^{13} \mathrm{C}$ NMR $\left(\mathrm{CDCl}_{3}, 100 \mathrm{MHz}\right): \delta=75.5,126.3,127.6,128.4,128.5$, $133.0,141.9,143.2$.

4-Methylphenyl-phenylmethanol $(6)^{2}$

Compound 6 was obtained as a white solid with up to $96 \%$ yield ( $48 \mathrm{mg}, 0.24 \mathrm{mmol}$ ).

${ }^{1} \mathrm{H} \operatorname{NMR}\left(\mathrm{CDCl}_{3}, 300 \mathrm{MHz}\right): \delta=[2.04(\mathrm{~s}, 1 \mathrm{H}, \mathrm{OH})], 2.31(\mathrm{~s}, 3 \mathrm{H}$, $\left.\mathrm{CH}_{3}\right), 5.76(\mathrm{~s}, 1 \mathrm{H}, \mathrm{CH}), 7.08-7.37\left(\mathrm{~m}, 9 \mathrm{H}, \mathrm{H}_{\mathrm{ar}}\right)$.

${ }^{13} \mathrm{C}$ NMR $\left(\mathrm{CDCl}_{3}, 75 \mathrm{MHz}\right): \delta=21.1,76.0,126.4,126.5,127.4$, $128.4,129.2,137.2,141.0,144.0$. 


\section{HPLC - separation conditions}

\section{4-Chlorophenyl-phenylmethanol (3) ${ }^{1}$}

Chiralcel OB-H, $30{ }^{\circ} \mathrm{C}, 230 \mathrm{~nm}, 90: 10$ heptane / i-PrOH, 0.5 $\mathrm{mL} / \mathrm{min} ; t_{\mathrm{R}}=25.7 \mathrm{~min}(R), 33.6 \mathrm{~min}(S)$.

\section{4-Methylphenyl-phenylmethanol $(6)^{2}$}

Chiralcel OD, $30{ }^{\circ} \mathrm{C}, 230 \mathrm{~nm}, 98: 2$ heptane / iPrOH, $0.9 \mathrm{~mL} / \mathrm{min}$; $t_{\mathrm{R}}=28.1 \mathrm{~min}(S), 31.3 \mathrm{~min}(R)$.

\section{1-Phenyl-propanol $(7)^{3}$}

Chiralcel OD, $20{ }^{\circ} \mathrm{C}, 254 \mathrm{~nm}, 98: 2$ heptane / i-PrOH, $0.5 \mathrm{ml} / \mathrm{min}$; $t_{\mathrm{R}}=15.5 \mathrm{~min}(R), 17.7 \mathrm{~min}(S)$.

\section{References}

1. Lee, J.-S.; Velarde-Ortiz, R.; Guijarro, A.; Wurst, J. R.; Rieke, R. D. J. Org. Chem. 2000, 65, 5428 .

2. Nakamura, S.; Oda, M.; Yasuda, H.; Toru, T. Tetrahedron 2001, 57, 8469 .

3. Bolm, C.; Schlingloff, G.; Harms, K. Chem. Ber. 1992, 125, 1191. 
Iागा

$\begin{array}{lllll}2.6 & 12.0 & 11.4 & 10.8 & \text { ppm }\end{array}$
RUDO PCL a4020331.b/h inova400/gas

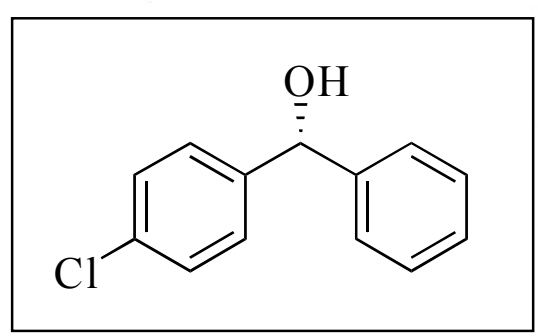




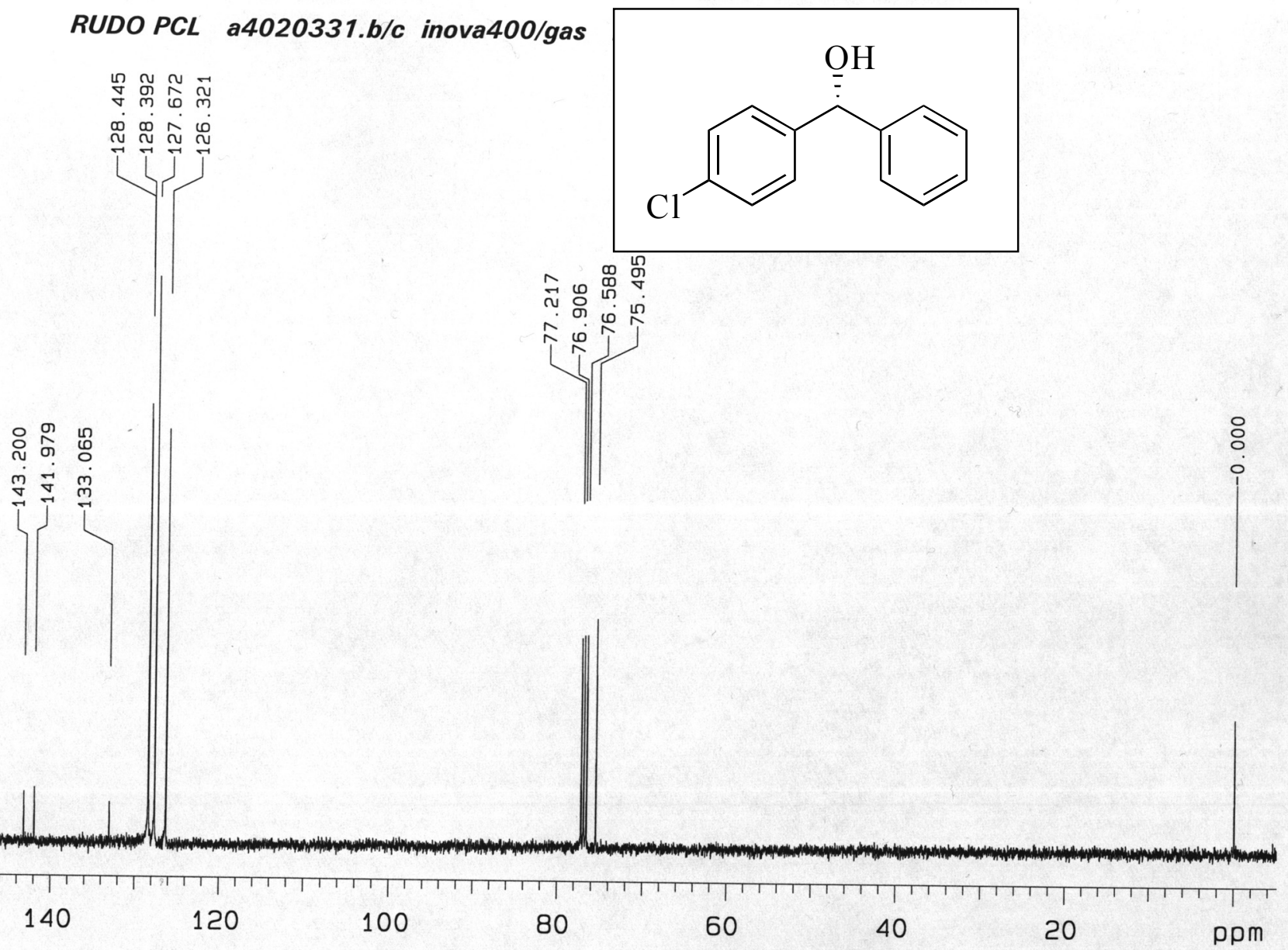

C. $\mathrm{CH} 2$ $\mathrm{CH}, \mathrm{CH}^{3}$ $\mathrm{CH}$ 


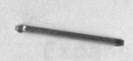

plot at $120 \mathrm{~Hz} / \mathrm{cm}: 0.40 \mathrm{ppm} / \mathrm{cm}$

ก

$\begin{array}{lllll}12.6 & 12.0 & 11.4 & 10.8 & \text { ppm }\end{array}$
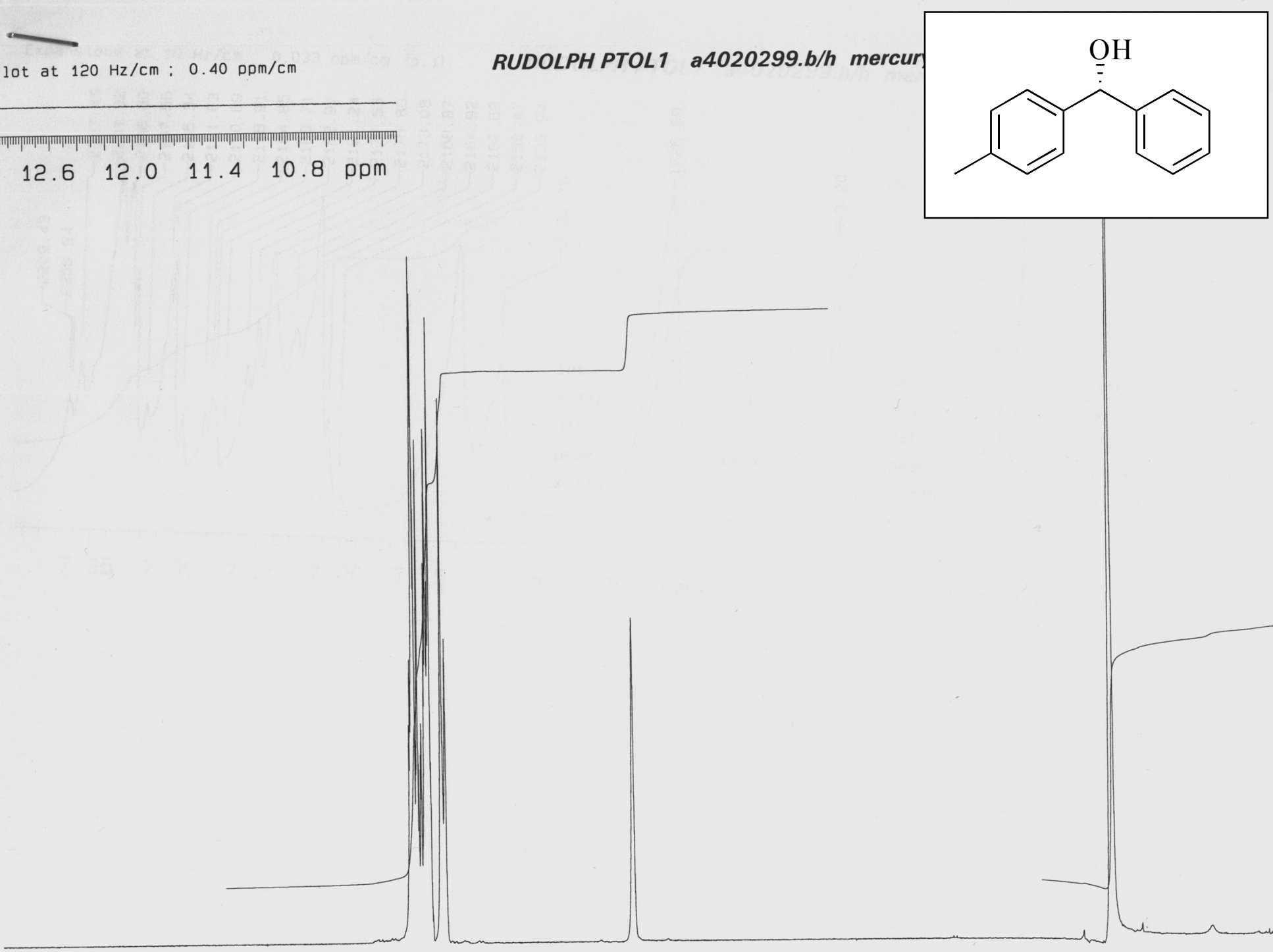

8

7

6

5

응
4

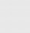


plot at $120 \mathrm{~Hz} / \mathrm{cm} ; 0.40 \mathrm{ppm} / \mathrm{cm}$

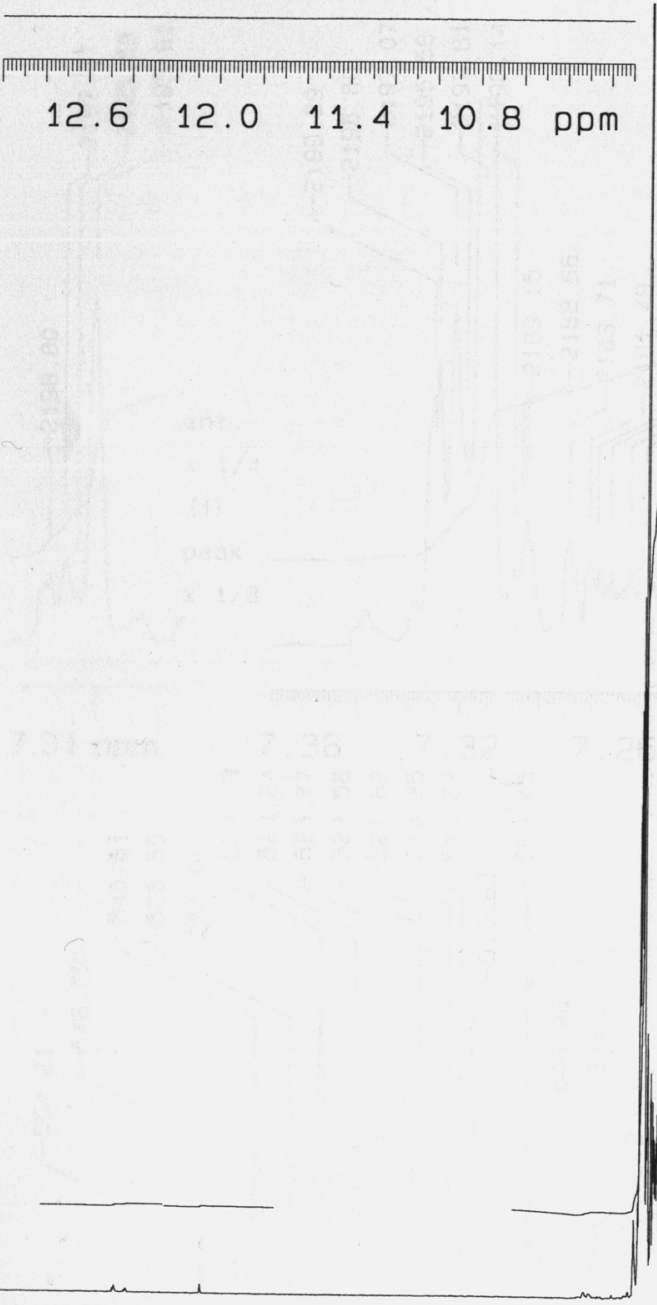

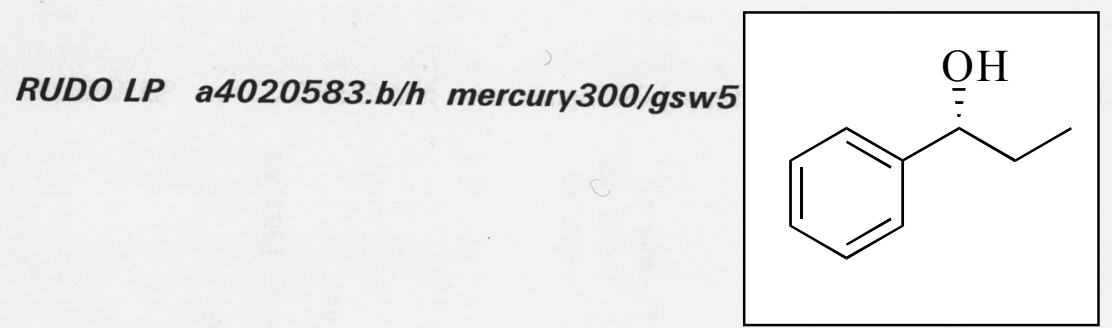

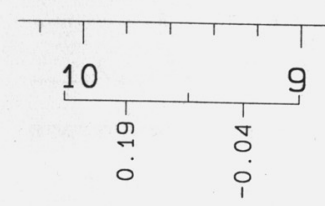

8

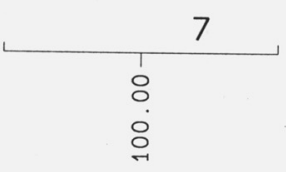

8 
RUDO LP a4020583.b/c mercury300/gsw5
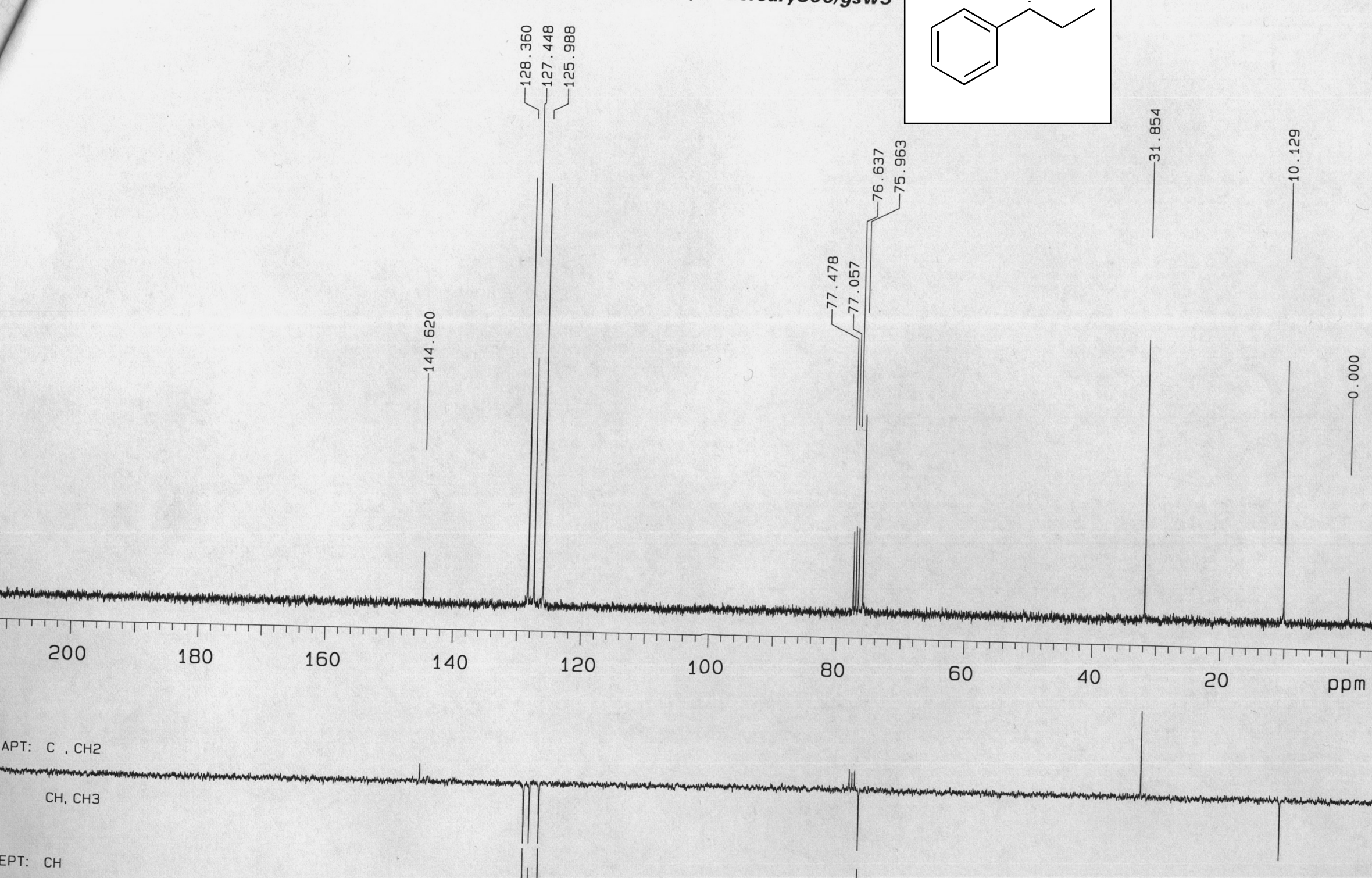